\title{
Retroperitoneal Air after ERCP with Sphincterotomy: Frequency and Clinical Significance
}

\section{-Retroperitoneal Air after Sphincterotomy}

\author{
Mario Anselmi Mendez ${ }^{1,2}$, José Gerardo Acosta Mata ${ }^{1}$, Carlos Flores Mladineo ${ }^{1}$, \\ Jaime Schwanner Carrasco ${ }^{1}$, Ana María Gemmato Pascazioo,2 \\ ${ }^{1}$ Endoscopic Surgery Teaching Care Unit, Guillermo Grant Benavente Hospital, Concepción, Chile \\ ${ }^{2}$ Department of Surgery Faculty of Medicine, University of Concepción, Concepción, Chile \\ Email: *manselmi@udec.cl
}

Received 11 December 2015; accepted 22 February 2016; published 25 February 2016

Copyright (C) 2016 by authors and Scientific Research Publishing Inc.

This work is licensed under the Creative Commons Attribution International License (CC BY). http://creativecommons.org/licenses/by/4.0/

\section{(c) (i) Open Access}

\begin{abstract}
Background and Aim: After successful medical management of a patient with a clinical picture suggestive of post-sphincterotomy duodenal perforation, in which a computerized axial tomography (CAT) scan of the abdomen revealed the presence of subcutaneous emphysema and retroperitoneal air, concern arose as to the frequency of pneumoretroperitoneum following endoscopic retrograde cholangiopancreatography (ERCP) with sphincterotomy (ES) and if any procedure dependent factors were associated with this problem. Aim: To assess the frequency and clinical significance of retroperitoneal air after endoscopic retrograde cholangiopancreatography with sphincterotomy. Methods: Fifty consecutive patients, who had undergone ERCP with sphincterotomy, were submitted to abdominal CT examinations within 24 hours after completion of the procedure. One patient was with a large precut, but a failed ERCP was also included. The ERCP findings were unknown to the radiologist. Results: Seven (14\%) of 50 patients showed CT findings of retroperitoneal air. All of them had uneventful post-procedural recovery. No clinical or laboratory abnormality was found in this group of patients. The presence of retroperitoneal air was not associated to the variables: precut, biliopancreatic disease type, endoscopic sphincterotomy length, additional endoscopic procedure (balloon exploration, gallstone extraction, stent insertion) or procedure duration. Conclusion: After ERCP with ES, retroperitoneal air is frequently found. In the absence of physical symptoms, retroperitoneal air is not clinically relevant and does not require specific treatment.
\end{abstract}

${ }^{*}$ Corresponding author.

How to cite this paper: Mendez, M.A., Mata, J.G.A., Mladineo, C.F., Carrasco, J.S. and Pascazio, A.M.G. (2016) Retroperitoneal Air after ERCP with Sphincterotomy: Frequency and Clinical Significance-Retroperitoneal Air after Sphincterotomy. Open Journal of Gastroenterology, 6, 31-38. http://dx.doi.org/10.4236/ojgas.2016.62005 
Keywords

Retroperitoneal Air, ERCP Complications, Duodenal Perforation

\section{Introduction}

In the hands of an expert, endoscopic retrograde cholangiopancreatography (ERCP) and endoscopic sphincterotomy (ES) are associated with high rates of success and few complications, most of which can be treated conservatively [1]. The most serious complications of ES are acute pancreatitis, bleeding, cholangitis, and perforation [2] [3]. The latter is infrequent, and its treatment ranges from simple conservative measures to immediate surgery [4] [5]. Stapfel [6] classified duodenal perforation, in descending order of severity, into four types, being type IV the finding of retroperitoneal air. However, this author did not refer to the incidence or clinical significance of such situations. To date, only one prospective study of 21 consecutive patients submitted to ES has been published. Retroperitoneal air was found in $29 \%$ of these [7].

In March 2005, our Endoscopic Surgery Teaching Care Unit treated an 89-year-old male patient with a clinical picture suggestive of acute cholangitis due to choledocholithiasis. The ERCP showed bile pus and the presence of multiple large stones. An ES was performed. The stones were partially removed and a stent was inserted, as it was not possible to obtain the cleaning of the bile duct.

In the period immediately following the procedure, it was verified the presence of extensive subcutaneous emphysema in the lumbar and thoracic region was associated with moderate intensity abdominal pain and fever. The patient remained hospitalized with a diagnosis of post-ERCP and sphincterotomy duodenal perforation. Radiology ruled out the presence of subphrenic air, however, a CAT scan of the abdomen revealed retroperitoneal air, but not collections or free fluid. Due to the discrete nature of the symptoms and the absence of signs of peritoneal irritation, leukocytosis, or hyperamylasemia, the patient was treated conservatively. He evolved with isolated low-grade fevers and gradual resolution of the subcutaneous emphysema. A week later, a duodenal leek was ruled out using upper gastrointestinal transit. Oral feeding was resumed and the patient was discharged from the hospital three days later remaining asymptomatic and after a three-week period was submitted to a new endoscopic procedure, resolving the choledocholitiasis completely. The post procedure course was satisfactory and was released from medical care nine months later.

Although the decision to treat this patient conservatively proved to be correct, the situation raised concern about the frequency with which retropneumoperitoneum is produced following ERCP with sphincterotomy and whether procedure dependent factors are associated with this problem.

Aim: To determine the frequency, cause, and eventual clinical relevance of the presence of retroperitoneal air following ERCP with sphincterotomy.

\section{Method}

Between January and November 2012 we studied 50 consecutive patients, submitted to ERCP with ES for the first time. Prior to take part in the study, written informed consent was obtained from each of them. Patients were excluded for any of the following reasons: pregnancy, previous sphincterotomy, Bilrroth I or II gastrectomy, ampullary cancer, pancreatic neoplasm or with duodenal infiltration.

The average age was $60 \pm 16.3$ years (range: 15 - 93 years), 37 (74\%) were female and $13(26 \%)$ males.

The gallbladder was in situ in 31 patients (62\%) and choledocolithiasis was found in 25 of these $(80.6 \%)$. The remaining 19 patients $(38 \%)$ had been submitted to previous cholecystectomy.

The main indication to perform the endoscopic procedure was the suspicion of common bile duct stones (44\%) the rest are shown in Table 1. The ERCP was performed with the patient in the prone position and 42 of them (84\%) underwent conscious sedation with Midazolam (1 - $5 \mathrm{mg}$ ), and hyoscine butylbromide (Buscapine up to $40 \mathrm{mg}$ ) was used as antiespasmodic. Anesthetic support was required in the remaining 8 cases (16\%), in 3 of these, due to severe sepsis and in the remaining to the advanced age and/or intercurrent illnesses.

In 10 cases $(20 \%)$, the duoendoscopy revealed the presence of a juxtapapillary diverticulum.

The ERCP and sphincterotomies were done using Olympus equipment, model TJF-160 (Olympus Optical Co., Tokyo, Japan). All the procedures were carried out by a surgeon endoscopist (MAM) and two residents of 
endoscopic surgery under strict supervision of the first.

The sphincterotomy was done using Olympus brand (Clever Cut 3V) or Wilson Cook (CT-20) instruments. In 10 patients $(20 \%)$, a precut was required in order to achieve the selective cannulation of the bile duct. The precuts were done using Olympus (KD 10Q) and Wilson Cook (HPC-2) needle sphincterotomes. Selective cannulation failed in one patient, but as this individual was submitted to a wide precut was included in the study.

Table 2 shows the cholangiographic diagnoses in the successfully cannulated patients $(n=49)$.

Endoscopic sphincterotomy was defined as the complete section of the ampulla of Vater and of the sphincter of Oddi. This was objectified endoscopically by observing the division of the sphincter muscle, the exit of a stream of bile, or by the easy passage of an inflated bile balloon through the incision. Table 3 shows the types of procedures performed.

Stones were removed with conventional Dormia baskets (Olympus FG-22Q). Large stones $(\mathrm{n}=12)$ were fragmented using Litho Crush (Olympus) in 8 and with Soehendra Lithotriptor (Wilson Cook) the remaining 4. For the management of stenosis $(n=3)$ polyethylene stents $(7$ and 10 French).were used.

CAT scans of the abdomen and pelvis were done between 1 and $24 \mathrm{~h}$ post-ERCP. The studies were carried out in our institution with a helical scan (Philips medical systems Netherlands, model Secura). Routine cuts of 7 $\mathrm{mm}$ thickness were made, with reconstruction of $7 \mathrm{~mm}$ and 1.5 pitch with a rotation of the gantry of 0.7 seconds, using $240 \mathrm{mAs}$ and $120 \mathrm{kVp}$. The sweep was done from the pulmonary bases to the perineum during a single apnea. No oral or intravenous contrast was used (Figure 1). The axial and reconstructed images were analyzed by two radiologists from the Imagenology Service. The presence of air and it location was recorded.

All patients were submitted to a clinical exam, and their amylase and lipase levels were measured immediately before and two hours after the procedure. Those in which there was suspicion of complication remained hospitalized until this was verified or ruled out. Patients were monitored until to 90 days post-procedure. The results data was prospectively stored in a specially designed form and tabulated in an Excel Spread sheet for later analysis. Results are shown as percentages and means with standard deviation.

Table 1. Indications for ERCP and sphincterotomy.

\begin{tabular}{ccc}
\hline Indication & $\mathbf{n = 5 0}$ & $\mathbf{\%}$ \\
\hline Choledocholithiasis & 22 & 44 \\
Acute biliary pancreatitis & 13 & 26 \\
Benign obstructive jaundice & 7 & 14 \\
Acute cholangitis & 5 & 10 \\
Malignant obstructive jaundice & 3 & 6 \\
\hline
\end{tabular}

Table 2. Cholangiography diagnoses in successfully cannulated patients.

\begin{tabular}{ccc}
\hline Diagnosis & $\mathbf{n = 4 9}$ & $\mathbf{\%}$ \\
\hline Choledocholithiasis & 31 & 63.3 \\
Acalculous bile duct & 13 & 26.5 \\
Malignant biliar duct stenosis & 4 & 8.2 \\
Juxtapapillary diverticulum & 3 & 6.1 \\
Mirizzi & 1 & 2 \\
Acute papillitis & 1 & 2 \\
\hline
\end{tabular}

Table 3. Types of endoscopic procedures performed.

\begin{tabular}{ccc}
\hline Procedure & $\mathbf{n = 5 0}$ & $\mathbf{\%}$ \\
\hline Sphincterotomy + Stone extraction & 26 & 52 \\
Sphincterotomy + balloon exploration & 15 & 30 \\
Sphincterotomy + drainage (stent) & 8 & 16 \\
Precut only & 1 & 2 \\
\hline
\end{tabular}




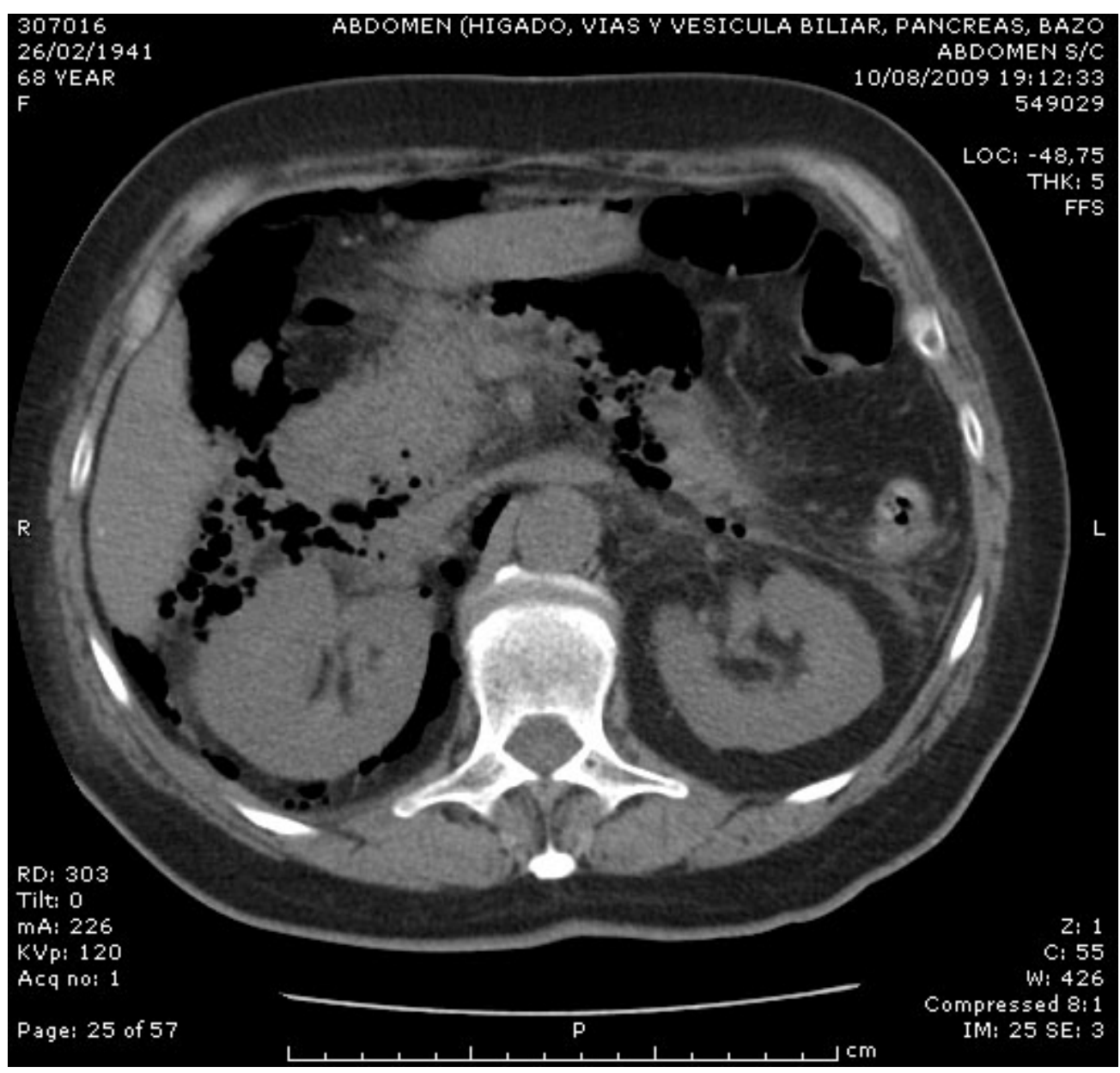

Figure 1. Early CAT scan of the abdomen following the procedure. This revealed an important amount of retroperitoneal air, but not collections or free fluid. The radiologist reported an Abdominal CT with signs that suggest a hollow viscera perforation with pneumoperitoneum and retroperitoneal air.

The research protocol was reviewed and approved by the Research and Ethical Commitees of the participant institutions.

\section{Results}

All the patients tolerated the procedure well and no complications occurred during endoscopy. Selective cannulations and the following sphincterotomies were achieved on the first attempt in 49 of the 50 patients (98\%), and in one (2\%), despite an extensive precut, it failed.

Complete sphincterotomy was done in 48 of the 50 patients studied (96\%). Of these, CAT scans revealed the presence of retroperitoneal air in only seven (14.6\%). In one patient (2\%), in whom a stent was inserted, the section of the sphincter was partial, and in the remaining one $(2 \%)$ only an extensive precut was performed. Although in this, patient the selective cannulation of the bile duct failed on the first attempt, a successful sphincterotomy was achieved 48 hours later.

The presence of retroperitoneal air was determined in seven of the 50 patients studied (14\%), all of whom had a satisfactory evolution and none of them showed any clinical or additional laboratory abnormality.

The mean duration of the endoscopic procedure was $30.1 \pm 13.3$ minutes (range: $10-60$ minutes) for those without retroperitoneal air and $35 \pm 11.2$ minutes (range: 20 - 50 minutes) for those found to have retropneumoperitoneum with a CAT scan. The procedures that lasted more than 30 minutes $(n=14)$, resulted in the presence or a retroperitoneal air in 3 patients $(21.4 \%)$.

Of the 10 patients submitted to a precut, only one (10\%) presented retroperitoneal air. Of the 10 patients with 
juxtapapillary diverticulum, the presence of air was found in one (10\%).

Only in 4 of the 31 cases with choledocholithiasis (12.9\%), the CAT scan showed the presence of air. A total of 12 patients had large stones ( $>15 \mathrm{~mm}$ in diameter). Of these, evidence of retroperitoneal air was found in two cases (16.7\%). Table 4 summarizes the association of these different factors with the presence of retroperitoneal air.

Other than aerobilia, the CAT scan did not reveal collections or other abnormalities. Three patients without signs of retroperitoneal air presented, as an early post-procedure finding, hyperamylasemia without clinical repercussions. The evolution was satisfactory in all cases.

Limitations: variables, including endoscopist skill and cannulation difficulty and sphicterotomy size, were not ease to measure.

\section{Discussion}

ERCP with ES is considered to be a safe and effective procedure for the treatment of a variety of biliopancreatic diseases. However, like any invasive technique, it is associated with morbidity; this rate is typically low but potentially serious [1].

In experienced hands, complications such as acute pancreatitis, bleeding and perforation are exceptional, but when they occur, they can put the life of the patient in danger [2] [3]. Perforation is a complication that occurs in less than $1 \%$ of patients undergoing endoscopic sphincterotomy, but may be associated with high mortality rates [5].

Currently, the incidence of perforation has decreased to less than 0.5 percent, probably associated to the improvement in experience and skill of endoscopists [8]. However, severe and fatal cases continue to occur [9] [10].

Patients with undetected leeks may present pain, fever, and leukocytosis up to hours after the procedure. Pneumomediastinum and subcutaneous emphysema can also appear [11]-[16].

Although rare, pneumothorax [17] and the presence of gas in the portal system [18] have also been described. Some publications report clinical cases in which patients develop retropneumoperitoneum, pneumoperitoneum, pneumomediastinum, subcutaneous emphysema, and even pneumothorax after the endoscopic procedure [17] [19] [20].

The diagnosis of perforation can often be suspected or made during the endoscopic procedure [5] [21], but is usually confirmed radiologically or by computed axial tomography, by demostrating open-air cavity or contrast extravasation. Often the physical exam can help assess the patient, but not all abdominal perforations present with an acute abdomen [12].

Stapfer [6] classified the duodenal perforation secondary to ERCP in decreasing order of severity into 4 types. He found that the mechanism of injury correlated well with radiological findings, and considering these two factors determine a good evidence for the indication for surgery.

Lateral or medial Wall perforations (Type I) caused by the endoscope, tend to be large in size and remote from the ampulla, and require inmediate surgery. Type I lesions often cause large, persistent contrast leaks in retroperitenal or intraperitoneal space.

Table 4. Different factors and presence of retroperitoneal air.

\begin{tabular}{cccc}
\hline Factor & $\mathrm{n}$ & Air $(+)$ & $\%$ \\
\hline ERCP + sphincterotomy & 49 & 7 & 14.3 \\
Procedure duration $<30 \mathrm{~min}$ & 36 & 4 & 11.1 \\
CBD stones removal & 26 & 3 & 11.5 \\
Use of biliary baskets & 22 & 3 & 13.6 \\
Juxtapapillary diverticulum & 20 & 1 & 5.0 \\
Procedure duration $>30$ min & 14 & 3 & 21.4 \\
Large stones removal & 12 & 2 & 16.7 \\
Precut & 10 & 1 & 10.0 \\
Use of balloon & 4 & 0 & 0 \\
\hline
\end{tabular}


Type II perforations are periduodenal which vary in severity, requiring surgery in up to $43 \%$ of patients in Stanfer's serie [6]. Type III is the perforation of the bile duct related to endoscopic instruments, such as guide wires or baskets. These are usually small. Type IV is the finding of isolated retroperitoneal air and its cause is attributed to intraduodenal air insufflation.

The management of secondary duodenal perforations following ES is controversial. While some suggest conservative treatment based on the patient's clinical course, others recommend surgical repair in all cases due to the complications associated with delayed intervention [5] [22]-[25]. In fact, Stepmfel et al. [6] suggests immediate surgical treatment only for type I perforations, which represent a serious complication associated with up to $25 \%$ mortality [21].

Perforations of types II and III, with minimal contrast extravasation and the absence of fluid collection, can be managed conservatively, without the need for surgery, but with close monitoring. Finally, type IV perforations are not considered to be true perforations and, thus, do not require surgical intervention.

For some years, it has been known that the continuous use of air to maintain the lumen of a hollow viscus distended, can lead to the presence of air inside or outside the walls of this, as is the case of colonic pneumatosis after trauma caused by gastrointestinal endoscopy, mucosal biopsy or polypectomy [26].

It seems evident that during ERCP with sphincterotomy and/or precut there are at least two factors that combine to the occurrence of retroperitoneal air. On one side they are the incisions made at the level of the ampulla and duodenal mucosa and on the other, the increased intraluminal pressure caused by insufflation of air required to maintain the distension of the duodenal lumen. In addition, brusque increments in intra-abdominal pressure such as those caused by coughing or nausea should also be considered. These often occur during or immediately after a procedure and can constitute an extra condition pushing air through the tissues and into the retroperitoneal space. This complication was described in a report of a clinical case following the forced dilatation of the sphincter of Oddi with a balloon for the removal of large stones [27]. However, since the ES to which this patient was submitted was described as "the most extensive possible", it is not clear whether the presence of retroperitoneal air was associated with the pneumatic dilatation, the ES, or both.

The purpose of the present study was to evaluate the frequency and significance of retroperitoneal air after ERCP with sphincterotomy. This survey was conducted by thin section computed tomography within 24 hours of the procedure.

Our study showed that the seven patients (14\%) found to have this condition evolved satisfactorily and none showed any clinical or additional laboratory abnormality. Moreover, although three of these patients presented elevated amylases due to acute biliary pancreatitis, the values of the pancreatic enzymes decreased after ES despite the finding of retroperitoneal air. Thus, there appeared to be no correlation between the presence of retropneumoperitoneum and hyperamylasemia.

It would seem logical to think that the larger the sphincterotomy, the more frequent the occurrence of retroperitoneal air. However, the results of this study did not show any relationship between the size of the sphincterotomy and retropneumoperitoneum. In fact, in our Endoscopic Unit, with few exceptions, this includes a complete section of both the ampulla of Vater and the sphincter of Oddi. However, only $14.3 \%$ of patients with complete sphincterotomy had retroperitoneal air. The results of this study do not support the association between the use of precut and increased rates of periampullary perforation [28]. In fact, in the present study, retroperitoneal air was found in only $10 \%$ of the patients submitted to a precut, this being even lower rate than that found for patients submitted to only ES.

The mean duration of the procedure in the 50 patients studied was $30.06 \pm 10.27$ minutes (range: 10 - 46 minutes). In 5 of the 7 patients with air, the duration was less than average and in $2(28.6 \%)$. This was exceeded, but the same thing happened in $11 / 43$ patients $(25.6 \%)$ without air. Therefore, this study showed no evidence supporting the claim the longer the duration of the procedure, the higher the retropneumoperitoneum rate.

The presence of a juxtapapillary diverticulum was found in one of the seven patients (14.3\%) with air and in six of the $42(14 \%)$ without air. Thus, unlike that suggested by Genzilinger [7], the presence of juxtapapillary diverticula does not seem to be associated with greater rates of retropneumoperitoneum. Moreover, a previous publication [29] showed that the presence of juxtapapillary diverticula is associated with lower rates of success in the cannulation of the bile duct, but once achieved, the ES and additional endoscopic procedures could be carried out successfully and safely in the vast majority of patients. This situation seems to be the same in relation to the presence of retroperitoneal air.

Chung et al. [24] indicated that the presence of air or retroperitoneal contrast was not predictive of surgery or 
related to the size of the perforation. Concluding that, given mild symptoms and rapid improvement with medical treatment, this complication could be treated satisfactorily without surgery. However, surgery should be performed if the pain and abdominal signals are prominent and do not improve after a short period of clinical management. The case of a free perforation of the peritoneal cavity is different [17].

\section{Conclusion}

Retroperitoneal air is a common finding of CAT scans following ERCP with sphincterotomy and can be caused by the amount of air used to distend the lumen of the duodenum. Retropneumoperitoneum is not related to the duration of the procedure, the length of the sphincterotomy, the presence of hyperamylasemia, or juxtapapillary diverticulum. The results of this study suggest that retroperitoneal air, in the absence of symptoms and physical signs, should not be the cause for alarm and does not require surgery.

\section{References}

[1] Anselmi, M., Cobo, R., Benavides, C. and Pacheco, F. (1998) Endoscopic Sphincterotomy: Indications, Successes, Failures and Complicatios. Revista Chilena de Cirugia, 50, 268-275.

[2] Freeman, M.L., Nelson, D.B., Sherman, S., et al. (1996) Complications of Endoscopic Sphincterotomy. The New England Journal of Medicine, 335, 909-918. http://dx.doi.org/10.1056/NEJM199609263351301

[3] Polydorou, A., Vezakis, A., Fragulidis, G., Katsarelias, D., Vagianos, C. and Polymeneas, G. (2011) A Tailored Approach to the Management of Perforations Following Endoscopic Retrograde Cholangiopancreatography and Sphincterotomy. Journal of Gastrointestinal Surgery, 15, 2211-2217. http://dx.doi.org/10.1007/s11605-011-1723-3

[4] Enns, R., Eloubeidi, M.A., Mergener, K., Jowell, P.S., Branch, M.S., Pappas, T.M. and Baillie, J. (2002) ERCP-Related Perforation: Risk Factors and Management. Endoscopy, 34, 293-298. http://dx.doi.org/10.1055/s-2002-23650

[5] Cotton, P.B., Lehman, G., Vennes, J., Geenen, J.E., Russell, R.C.G., Meyers, W.C., Liguory, C. and Nickl, N. (1991) Endoscopic Sphincterotomy Complications and Their Management: An Attempt to Consensus. Gastrointestinal Endoscopy, 37, 383-393. http://dx.doi.org/10.1016/S0016-5107(91)70740-2

[6] Stapfer, M., Selby, R.R., Stain, S.C., Katkhouda, N., Parekh, D., Jabbour, N. and Garry, D. (2000) Management of Duodenal Perforations after Endoscopic Retrograde Cholangiopancreatography and Sphincterotomy. Annals of Surgery, 232, 191-198. http://dx.doi.org/10.1097/00000658-200008000-00007

[7] Genzlinger, J.L., McPhee, M.S., Fisher, J.K., et al. (1999) Significance of Retroperitoneal Air after Endoscopic Retrograde Cholangiopancreatography with Sphincterotomy. The American Journal of Gastroenterology, 94, 1267-1270.

[8] Rabenstein, T., Schneider, H.T., Hahn, E.G. and Ell, C. (1998) 25 Years of Endoscopio Sphincterotomy in Erlangen: Assessment of the Experience in 3498 Patients. Endoscopy, 30, A194-A201. http://dx.doi.org/10.1055/s-2007-1001439

[9] Pierie, J.P. and van Vroonhoven, T.J. (1999) Obstructive Cholangiopathy: Are Endoscopic Retrograde Cholangiopancreatography and Papillotomy Always Really Necessary? Ned Tijdschr Geneeskd, 143, 1497-1499.

[10] Vandervoort, J., Soetikno, R.M., Tham, T.C., et al. (2002) Risk Factors for Complications after Performance of ERCP. Gastrointestinal Endoscopy, 56, 652-656. http://dx.doi.org/10.1016/S0016-5107(02)70112-0

[11] Ciaccia, D., Branch, M.S. and Baillie, J. (1995) Pneumomediastinum after Endoscopic Sphincterotomy. The American Journal of Gastroenterology, 90, 475-477.

[12] Colemont, L.J., Pelckmans, P.A., Moorkens, G.H. and Van Maercke, Y.M. (1988) Unilateral Periorbital Emphysema: An Unusual Complication of Endoscopic Papillotomy. Gastrointestinal Endoscopy, 34, 473-475. http://dx.doi.org/10.1016/S0016-5107(88)71440-6

[13] Tam, F., Prindiville, T. and Wolfe, B. (1989) Subcutaneous Emphysema as a Complication of Endoscopic Sphincterotomy of the Ampulla of Vater. Gastrointestinal Endoscopy, 35, 447-449. http://dx.doi.org/10.1016/S0016-5107(89)72857-1

[14] Scarlett, P.Y. and Falk, G.L. (1994) The Management of Perforation of the Duodenum Following Endoscopic Sphincterotomy: A Proposal for Selective Therapy. Australian and New Zealand Journal of Surgery, 64, 843-846. http://dx.doi.org/10.1111/j.1445-2197.1994.tb04561.x

[15] Doerr, R.J., Kulaylat, M.N., Booth, F.V. and Corasanti, J. (1996) Barotrauma Complicating Duodenal Perforation during ERCP. Surgical Endoscopy, 10, 349-351. http://dx.doi.org/10.1007/BF00187390

[16] Alexiou, K., Sakellaridis, T., Sikalias, N., Karanikas, I., Economou, N. And Antsaklis, G. (2009) Subcutaneous Emphysema, Pneumomediastinum and Pneumoperitoneum after Unsuccessful ERCP: A Case Report. Cases Journal, 2, 120. http://dx.doi.org/10.1186/1757-1626-2-120 
[17] Savides, T., Sherman, S., Kadell, B., et al. (1993) Bilateral Pneumothoraces and Subcutaneous Emphysema after Endoscopic Sphincterotomy. Gastrointestinal Endoscopy, 39, 814-881. http://dx.doi.org/10.1016/S0016-5107(93)70273-4

[18] Merine, D. and Fishman, E.K. (1989) Uncomplicated Portal Venous Gas Associated with Duodenal Perforation Following ERCP: CT Features. Journal of Computer Assisted Tomography, 13, 138-139. http://dx.doi.org/10.1097/00004728-198901000-00033

[19] Ferrara, F., Luigiano, C., Billi, P., et al. (2009) Pneumothorax, Pneumomediastinum, Pneumoperitoneum, Pneumoretroperitoneum, and Subcutaneous Emphysema after ERCP. Gastrointestinal Endoscopy, 69, 1398-1401. http://dx.doi.org/10.1016/j.gie.2008.08.004

[20] Song, S.Y., Lee, K.S., Na, K.J. and Ahn, B.H. (2009) Tension Pneumothorax after Endoscopic Retrograde Pancreatocholangiogram. Journal of Korean Medical Science, 24, 173-175. http://dx.doi.org/10.3346/jkms.2009.24.1.173

[21] Bell, R.C.W., Van Stiegmann, G., Goff, J., et al. (1991) Decision for Surgical Management of Perforation after Endoscopic Sphincterotomy. The American Journal of Surgery, 57, 237-240.

[22] Raju, G.S. and Gajula, I. (2004) Endoclips Por GI Endoscopy. Gastrointestinal Endoscopy, 59, 267-279. http://dx.doi.org/10.1016/S0016-5107(03)02110-2

[23] Dunham, F., Bourgeois, N., Gelin, M., Jeanmart, J., Toussaint, J. and Cremer, M. (1982) Retroperitoneal Perforation Following Endoscopic Sphincterotomy; Clinical Course and Management. Endoscopy, 14, 92-96. http://dx.doi.org/10.1055/s-2007-1021589

[24] Chung, R.S., Sivak, M.V. and Ferguson, D.R. (1993) Surgical Decisions in the Management of Duodenal Perforation Complicating Endoscopic Sphincterotomy. The American Journal of Surgery, 165, 700-703. http://dx.doi.org/10.1016/S0002-9610(05)80791-3

[25] Chaudhary, A. and Aranya, R.C. (1996) Surgery in Perforation after Endoscopic Sphincterotomy: Sooner, Later or Not at All? Annals of the Royal College of Surgeons of England, 78, 206-208.

[26] Heer, M., Altorfer, J., Pirovino, M. and Schmid, M. (1983) Pneumatosis Cystoides Coli: A Rare Complication of Colonoscopy. Endoscopy, 15, 119-120. http://dx.doi.org/10.1055/s-2007-1021484

[27] Katsinelos, P., Chatzimavroudis, G., Pipilidis, I., Zavos, C., Lazaraki, G., Tzilves, D., Paroutoglou, G. and Kountouras, J. (2008) Benign Retroneumoperitoneum Development after Endoscopic Sphicterotomy and Large Balloon Dilation of Biliary Sphincter for Removal of Large Biliary Stones: A Case Report. Cases Journal, 1, 279. http://dx.doi.org/10.1186/1757-1626-1-279

[28] Sarli, L., Porrini, C., Costi, R., Regina, G., Violi, V., Ferro, M. and Roncoroni, L. (2007) Operative Treatment of Periampullary Retroperitoneal Perforation Complicating Endoscopic Sphincterotomy. Surgery, 142, 26-32. http://dx.doi.org/10.1016/j.surg.2007.02.002

[29] Anselmi, M. and Arancibia, S. (2001) Significance of Duodenal Yuxtapapilary Diverticulum in the Diagnosis and Endoscopic Treatment of Biliopancreatic Pathology. Revista Chilena de Cirugia, 53, 434-437. 